\title{
POTENCIAL EMPRENDEDOR DE LOS ESTUDIANTES DE LA ESPECIALIDAD DE NEGOCIOS INTERNACIONALES DE LA UNMSM
}

\author{
Emma Emilia Pérez Palacios (*)
}

E-mail: eeperezpalacios@yahoo.com

\begin{abstract}
RESUMEN
La presente investigación tiene por finalidad el estudio de la conducta emprendedora desde la perspectiva de los valores, basada en la teoría de Schwartz que sostiene que los valores influyen en la decisión de las personas de formar una empresa. Es una investigación de tipo descriptivo explicativo aplicada a los estudiantes de la especialidad de Negocios Internacionales de la Universidad Nacional Mayor de San Marcos. La metodología utilizada para el tratamiento de los datos es de carácter descriptivo e inferencial. El resultado de la investigación muestra que existe una relación directa entre la capacidad emprendedora y los valores de los estudiantes quienes, a su vez, se caracterizan porque disfrutan de lo que hacen, tienen independencia en el pensamiento, eligen sus propias metas, y cuentan con familiares empresarios que influyen significativamente en su decisión de formar empresas, por lo que podemos concluir que ellos poseen características emprendedoras que necesitan ser reforzadas para que en el futuro se conviertan en empresarios de éxito.
\end{abstract}

Palabras clave: Conducta emprendedora, negocios internacionales

\section{ABSTRACT}

The present investigation has as an aim the study of the enterprising attitude from the point of view of the values, cradle in the theory of Schwartz, that maintains that the values influence in the decision of the people when forming a company. It is an investigation of descriptive type - explanatory applied to the students of the specialty of International Businesses of the Universidad Nacional Mayor de San Marcos. The methodology used for the treatment of the data is of descriptive and inferencial character. The result of the investigation sample that exists a direct relation between the enterprising capacity and the values of the students, as well, is characterized because they enjoy and so they do, have independence in the thought, choose his own goals; having familiar businessmen influences significantly in its decision to form companies, reason why we can conclude that they own enterprising characteristics that need to be reinforced so that the future they become success businessmen.

Key words: Entenprising actitude, international businesses

(*) Licenciada en Estadística por la Universidad Nacional Federico Villarreal. Profesora Auxiliar de la Facultad de Ciencias Administrativas de la UNMSM. 


\section{INTRODUCCIÓN}

En el Perú diversos factores han creado un clima propicio para el emprendimiento: mercados emergentes en Lima y el interior del país, así como exportaciones crecientes y expectativas de mayores beneficios por los tratados de libre comercio, factores que han generado un mayor interés por la creación de nuevas empresas.

En el siglo pasado, a fines de los años 80 y mediados de los 90, hubo un significativo crecimiento de la participación del autoempleo en la Población Económicamente Activa (PEA) ocupada. Simultáneamente, también creció el empleo en microempresas.

Según datos proporcionados por la Encuesta Nacional de Hogares (ENAHO), en 1995 el 59.1\% de la PEA urbana estaba ocupada en microempresas; en 1997, el 59.6\% y en el año 1999 la proporción se incrementó hasta $63.3 \%$ de la PEA urbana. Estas cifras han llevado a más de un analista a sugerir que la solución al problema del empleo, entendido como la falta de puestos de trabajo, se encuentra en la micro y pequeña empresa. Por otro lado, su vinculación con la problemática laboral es directa ya que un significativo número de jóvenes trabajan en una microempresa u otros optan por el autoempleo, siendo común encontrar a un joven trabajando por su propia cuenta, de manera independiente.

El autoempleo resulta ser una opción importante, dadas las dificultades de inserción laboral de los jóvenes. El tema de desempleo de los jóvenes es materia de preocupación entre los gobiernos de países desarrollados y no desarrollados, tanto por el potencial de recursos humanos desperdiciados como por sus efectos colaterales de frustración y su potencial secuela de manifestaciones antisociales. En nuestro país, el problema se torna más complejo debido a su vínculo con mecanismos que tienden a perpetuar la pobreza. Las universidades, tanto nacionales como privadas, juegan un rol importante en la solución del problema, impartiendo conocimientos a sus jóvenes estudiantes para que superen su situación económica, generando sus propios puestos de trabajo.

El estudio de las capacidades emprendedoras es una línea de investigación de gran interés dada la actual situación socioeconómica, que tiene como objetivo que los estudiantes abran nuevos caminos empresariales y sean capaces de aprovechar las oportunidades de un mercado globalizado, además de contribuir al desarrollo económico local, regional y nacional.

La posibilidad que una persona acceda a un empleo depende de las características del individuo, del mercado de trabajo y de las técnicas y procesos de inserción. Sin embargo, la persona emprendedora no se limita a la forma convencional de incorporación al mercado laboral sino que es ella quien crea su propio empleo y, a su vez, da empleo a otros.

La Universidad Nacional Mayor de San Marcos a través de su Escuela Profesional de Negocios Internacionales no es ajena a este problema. Ha asumido como un reto el desarrollo emprendedor de sus estudiantes de un modo integral donde no sólo se toman en cuenta los rasgos de personalidad sino también los valores que marcarán su manera de actuar, aprovechando las lecciones derivadas de las investigaciones y la docencia, traduciéndolas en planteamientos específicos orientados al desarrollo sostenible del país.

En la presente investigación se realizó un diagnóstico de las capacidades emprendedoras de los estudiantes de la especialidad de Negocios Internacionales de la Universidad Nacional Mayor de San Marcos, explorando los rasgos de la personalidad y los valores personales dentro de las dimensiones del individualismo y colectivismo. Esto nos conduce a plantearnos las siguientes interrogantes:

\section{Problemática}

a) Problema general. ¿Existe relación entre los niveles de emprendedorismo y los valores personales de los jóvenes estudiantes de la especialidad de Negocios Internacionales de la Universidad Nacional Mayor de San Marcos?

b) Problemas específicos. ¿Cuáles son los niveles de emprendedorismo que prevalecen en los estudiantes de la especialidad de Negocios Internacionales de la UNMSM?

¿Cuáles son los valores que prevalecen en los estudiantes de la especialidad de Negocios Internacionales de la UNMSM?

¿Los valores personales individualistas se relacionan en forma directa con los niveles de emprendedorismo de los estudiantes de la especialidad de Negocios Internacionales de la UNMSM?; a su vez, ¿los valores colectivistas se relacionan en forma inversa con los niveles de emprendedorismo? 
¿Cuáles son las condiciones sociodemográficas que presentan diferencias significativas en los niveles de emprendedorismo y los valores personales de los estudiantes de la especialidad de Negocios Internacionales de la UNMSM?

\section{Objetivos de la investigación}

a) Objetivo general. Identificar la relación existente entre los niveles de emprendedorismo y los valores personales de los estudiantes de la especialidad de Negocios Internacionales de la UNMSM.

\section{b) Objetivos específicos.}

- Conocer los niveles de emprendedorismo de los estudiantes de la especialidad de Negocios Internacionales de la UNMSM.

- Determinar los valores que prevalecen en los estudiantes de la especialidad de Negocios Internacionales de la UNMSM.

- Determinar si los valores personales individualistas se relacionan en forma directa con los niveles de emprendedorismo, y si los valores colectivistas se relacionan en forma inversa con los niveles de emprendedorismo de los estudiantes de la especialidad de Negocios Internacionales de la UNMSM.

- Identificar las diferencias existentes entre los niveles de emprendedorismo y los valores personales según las condiciones sociodemográficas de los estudiantes de la especialidad de Negocios Internacionales de la UNMSM.

\section{Justificación}

A través de la investigación conoceremos las características emprendedoras de los estudiantes de la especialidad de Negocios Internacionales. Por medio de la teoría de Schwartz conoceremos los valores que conducen a una actitud emprendedora en los estudiantes para que en el futuro se conviertan en empresarios de éxito.

\section{Importancia}

La presente investigación es de gran importancia debido a que, en la mayoría de los programas formativos para jóvenes emprendedores, brindan cursos que permiten desarrollar habilidades para llegar a ser un buen emprendedor, tales como organización, liderazgo, negociación, creatividad, innovación, entre otros, y no prestan importancia a inculcar una serie de valores que van a servir de guía a la persona que tiene el deseo de formar una empresa. Por tanto, sería importante que la formación en valores se incluya en la currícula, y que sea considerada como política de la universidad. De esta manera se logrará que una persona emprendedora sea capaz de fomentar valores que permitan alcanzar una relación directa con la sociedad, y así promover el desarrollo y consolidación de empresas en nuestro país.

\section{Limitaciones de la Investigación}

La presente investigación se delimitó en los siguientes aspectos:

a) Delimitación espacial. El trabajo de investigación se desarrolló a nivel de estudiantes de los ciclos impares de la carrera: primero, tercero, quinto, séptimo y noveno ciclo de la especialidad de Negocios Internacionales de la Universidad Nacional Mayor de San Marcos, durante el período académico 2007-I.

b) Delimitación temporal. El periodo en el cual se desarrolló la investigación corresponde al primer semestre académico del año 2007.

\section{MÉTODOS}

Por el enfoque cuantitativo, la presente investigación es un estudio descriptivo - explicativo.

Es descriptivo porque a partir de una muestra probabilística de estudiantes de la Escuela de Negocios Internacionales de la UNMSM se descubren características emprendedoras y se comprueba una asociación entre las variables de la población. En la investigación descriptiva se recolecta información relevante de muestras con respecto a un mismo fenómeno; en base a la comparación de los datos recopilados, es posible realizar una comparación entre ellos ${ }^{1}$.

"La investigación explicativa es el tipo de investigación más usada en las Ciencias Sociales. Cuando en una investigación el investigador se plantea como objetivos estudiar el por qué de las cosas, hechos, fenómenos o situaciones, a estas investigaciones se les denomina explicativas".

1 Citado en Sánchez C., Hugo y Reyes M., Carlos. (1985). Pág. 62.

2 Hernández (1996). Citado en Bernal T. César Augusto. (2000). Capítulo 7, pág. 113. 
"Cuanto mayor número de variables estén correlacionadas en el estudio y mayor sea la fuerza de las relaciones, más completa será la explicación"3.

La población está conformada por los estudiantes de la Escuela de Negocios Internacionales de la UNMSM de primer, tercero, quinto, séptimo y noveno grado académico o ciclo, matriculados en el semestre 2007-I, los que son 605 alumnos y alumnas, en promedio.

El tamaño de la muestra se determinó mediante muestreo aleatorio estratificado por ciclos. Para determinar el número de estudiantes que formaron parte de la muestra, se utilizó la siguiente fórmula de Sierra Bravo para poblaciones finitas:

$n=\frac{Z^{2} \times P \times Q \times N}{E^{2}(N-1)+Z^{2} \times P \times Q}$

Donde:

$E=5 \%$. Margen de error.

Proporción o probabilidad de éxito: 0.50

Nivel de confianza del 95\% $(Z=1.96)$

$\mathrm{N}=605$

Reemplazando valores en la fórmula tenemos:

$$
\begin{aligned}
& n=\frac{(1.96)^{2} \times 0.50 \times 0.50 \times 605}{(0.05)^{2} \times(605-1)+(1.96)^{2} \times 0.50 \times 0.50}= \\
& =\frac{581.042}{2.4704}=235.20
\end{aligned}
$$

Entonces:

$\mathrm{n}=235$ alumnos

\section{Distribución de la muestra en los estratos: Ciclos}

Se repartió el tamaño de muestra "n" en forma proporcional al tamaño de los estratos de la población. La fórmula usada es la siguiente:

$$
n_{i}=n \frac{N_{i}}{N} \quad \operatorname{Con} i=1,2,3, \ldots
$$

Donde:

$\mathrm{Ni}=$ Tamaño del estrato $\mathrm{i}$.

$\mathrm{N}=$ Tamaño de la población.

$\mathrm{n}=$ Tamaño de la muestra.

$r=\quad$ Número de estratos.
El tamaño de la muestra fue de 235 alumnos cantidad que se reparte en forma proporcional entre los estratos.

Para el primer estrato, reemplazando valores en la fórmula, tenemos:

$$
n_{i}=n \frac{N_{i}}{N}=235 \frac{115}{605}=46.66 \approx 48
$$

De esta forma se distribuye la muestra para los siguientes estratos. A continuación presentamos el cuadro de la distribución de la muestra en cada uno de los estratos (ver Cuadro $\mathrm{N}^{\circ} 1$ ):

Cuadro № 1

Distribución de la muestra entre los estratos

\begin{tabular}{|c|c|c|}
\hline Ciclos & N & n \\
\hline I & 115 & 45 \\
\hline III & 187 & 72 \\
\hline V & 124 & 48 \\
\hline VIII & 103 & 40 \\
\hline IX & 76 & 30 \\
\hline Total & $\mathbf{6 0 5}$ & $\mathbf{2 3 5}$ \\
\hline
\end{tabular}

\section{Selección de las Unidades Muestrales}

Se realizó a través de muestreo PPT (probabilidad proporcional al número de alumnos por sección) en cada uno de los ciclos. Para el caso de la selección de una sección por ciclo, se eligieron a los estudiantes de las secciones con muestreo aleatorio simple. Para el caso de dos o tres secciones por ciclo se asignó la muestra entre las secciones en forma proporcional y luego, entre los estudiantes que conformaron la muestra, se realizó la selección aleatoria.

\section{Instrumentos}

El cuestionario estuvo conformado por tres partes: la primera correspondiente a datos de identificación general; la segunda, identificación de los rasgos personales de los estudiantes (tomado del Programa Escuela Emprende 2004), y la tercera parte estuvo conformada por preguntas del cuestionario de valores de Schwartz de 1994. Dichos aspectos se describen a continuación.

a) Datos de identificación general. En la primera parte del cuestionario se recopilan datos sobre aspectos generales del estudiante en 16 preguntas tales como: ciclo de estudios, sexo,

3 Citado en Hernández, Roberto; Fernández, Carlos y Baptista, Pilar. (1991). Capítulo 4, pág. 66. 
edad, lugar de nacimiento, distrito de residencia, lugar de nacimiento del padre y madre, familiar con negocio propio, parentesco con la persona que tiene el negocio, condición laboral, ocupación, experiencia empresarial, éxito de la empresa, si piensa formar una empresa en el futuro y medidas iniciadas para llevar adelante su empresa.

b) Cuestionario: Evaluación de los Rasgos Personales del Ministerio de Trabajo. Programa Escuela Emprende (2004). La prueba consiste en 32 pares de oraciones. En cada par, el alumno debió escoger una oración e indicar si la respuesta es $\mathrm{A}$ o $\mathrm{B}$.

Las preguntas deben agruparse en seis factores relacionados a los rasgos personales de los emprendedores:

- Creatividad e innovación.

- Locus de control interno.

- Proactividad e iniciativa.

- Perseverancia.

- Autoeficacia general.

- Disposición de asumir riesgos.

La muestra de estudiantes se clasificó en dos grupos: alto y bajo nivel de emprendedorismo, con la finalidad de realizar comparaciones entre los estudiantes con las variables de la investigación. Se utilizó como criterio para esta división la puntuación media.

c. Cuestionario: Perfil de valores motivacionales de Schwartz (1994). El cuestionario consta de 40 preguntas agrupadas de la siguiente forma (ver Cuadro $\mathrm{N}^{\circ} 2$ ):

Cuadro $\mathrm{N}^{\circ} 2$

Cuestionario agrupado por factores

\begin{tabular}{|l|l|}
\hline Factores & Pregunta № \\
\hline Conformidad & $7,16,28,36$ \\
\hline Tradición & $9,20,25,38$ \\
\hline Benevolencia & $12,18,27,33$ \\
\hline Universalismo & $3,8,19,23,29,40$ \\
\hline Autodirección & $1,11,22,34$ \\
\hline Estimulación (Motivación) & $6,15,30$ \\
\hline Hedonismo & $10,26,37$ \\
\hline Logro & $4,13,24,32$ \\
\hline Poder & $2,17,39$ \\
\hline Seguridad & $5,14,21,31,35$ \\
\hline
\end{tabular}

Los ítems fueron calificados sobre la base de una escala de Likert de seis puntos, de la siguiente forma:

- No se parece nada a mí: 1 punto

- No se parece a mí. 2 puntos

- Se parece poco a mí: 3 puntos

- Se parece algo a mí: 4 puntos

- Se parece a mí: 5 puntos

- Se parece mucho a mí: 6 puntos

\section{Procedimiento de recolección de datos.}

La recolección de información se realizó en el transcurso de una semana por la autora de la investigación.

\section{Técnicas de análisis de datos.}

Se utilizaron las siguientes herramientas estadísticas:

- Media aritmética, para identificar los rasgos de emprendedorismo y los valores motivacionales de mayor preferencia de los estudiantes.

- Correlaciones entre las variables para identificar las relaciones existentes entre las variables.

- Tablas y gráficos de las variables sociodemográficas para realizar un análisis de los datos de la muestra.

- Correlación Item total corregida y Alfa de Cronbach para realizar la confiabilidad de las preguntas de los cuestionarios con escala de Likert.

- Análisis factorial exploratorio para analizar si las variables formarán grupos en los seis factores evaluados para identificar los rasgos personales con actitud emprendedora, y de esta forma comprobar la validez del instrumento.

- Análisis factorial confirmatorio para evaluar la validez del cuestionario de valores. De esta forma se confirmó que la estructura propuesta por Schwartz se mantiene con los datos recopilados.

- La prueba "T" de Student sobre dos muestras independientes para comparar si existían diferencias estadísticamente significativas entre las variables sociodemográficas con dos niveles. 
- Análisis de varianza para analizar las diferencias estadísticas significativas para variables con más de dos niveles.

\section{Segunda parte del instrumento: Cuestionario para la Evaluación de los Rasgos Personales del Ministerio de Trabajo. Programa Escuela Emprende (2004)}

a) Validez del instrumento. Para comprobar la validez del cuestionario resultó indispensable realizar el análisis factorial exploratorio de los datos con la finalidad de establecer cuál sería la estructura factorial que existía entre los datos de la muestra con las dimensiones propuestas. El análisis factorial exploratorio fue realizado sobre la base del procedimiento de los componentes principales, utilizándose el método de rotación oblicua Promax, debido a que se esperaba encontrar que los factores que se constituyen en el instrumento fueran dependientes entre sí. Los resultados muestran la existencia de seis factores relevantes, lo que permite explicar el $34.19 \%$ de la varianza total.

b) Confiabilidad. Con la estructura encontrada de los seis factores se procedió a verificar si las preguntas cumplían con los niveles de confiabilidad en cada uno de los factores.

7. Tercera parte del instrumento: Cuestionario: Perfil de valores motivacionales de Schwartz (1994)

a) Validez. La validez de constructo se estudió por medio del análisis factorial confirmatorio, a través del cual fue posible establecer si la estructura teórica latente ya establecida se encuentra presente en el conjunto de datos, tratando de determinar si los 10 factores (valores motivacionales) obtenidos en la versión original se mantenían en los datos de la muestra evaluada.

Se analizó el ajuste global del modelo propuesto por Schwartz para comprobar si es adecuada la representación del conjunto completo de relaciones causales.

Las tres medidas del ajuste absoluto son: el ratio de verosimilitud de la prueba chi-cuadrada $\left(X^{2}\right)$, el índice de bondad de ajuste $(\mathrm{GFI})$ y la raíz cuadrada de la media de los residuos al cuadrado (RMSR).

Según la tabla, el valor de la chi-cuadrada es de 185.27 con 28 grados de libertad; esta- dísticamente es significativo para el nivel de significancia 0.000 . Dado que la sensibilidad de esta medida está afectada por el tamaño de la muestra $\mathrm{n}=235$, se concluye que existen diferencias significativas.

El valor GFI es de 0.96 que es un nivel aceptable como lo es también el valor de RMSR de 0.053; por lo tanto, las medidas del ajuste absoluto indican que el modelo es aceptable.

Al comparar el resultado chi-cuadrado del modelo propuesto por Schwartz con el modelo independiente, se observa que el chi-cuadrado del modelo de Schwartz es menor que el chicuadrado del modelo independiente (185.27 $<3714.41)$.

El índice de bondad de ajuste (AGFI), el índice de Tucker - Lewis (TLI) y el índice de ajuste normado (NFI) caen por encima del umbral deseado de 0.90. Se concluye que el modelo propuesto de Schwartz con los datos de la muestra es mejor que el modelo independiente, con lo que se comprueba la validez del modelo.

b) Confiabilidad. Una vez realizada la validez el segundo paso es necesario analizar si el cuestionario es confiable; se ha buscado la consistencia interna de las 40 preguntas agrupadas en las 10 variables de la escala de medición propuesta por Schwartz. La confiabilidad es la consistencia interna que se aplica a la consistencia entre las variables en una escala; en este caso, el promedio. Se parte de la premisa siguiente: para que se cumpla la consistencia interna, las escalas relacionadas a un grupo de preguntas deberían estar midiendo las mismas construcciones y, de esta forma, estarían altamente correlacionados.

En conclusión, la estructura factorial encontrada en el modelo propuesto ha demostrado la presencia de tres factores asociados entre sí, que cumplen con la definición conceptual propuesta por Schwartz y que, además, presentan validez y confiabilidad; por tal motivo, el desarrollo del presente estudio se realizó en base a la escala de puntaciones promedios.

\section{RESULTADOS}

De una muestra de 235 estudiantes de la Escuela de Negocios Internacionales de la Universidad Nacional Mayor de San Marcos, el 19.2\% corres- 
ponde a estudiantes del primer ciclo, el $30.6 \%$ a estudiantes del tercer ciclo, el $20.4 \%$ pertenece a estudiantes del quinto ciclo, el $17 \%$ a estudiantes del séptimo ciclo y el $12.8 \%$ a estudiantes del noveno ciclo (ver Gráfico $\mathrm{N}^{\circ} 1$ ).

Del 100\% de los encuestados, el $62.1 \%$ son mujeres y el $37.9 \%$ son hombres (ver Gráfico $\mathrm{N}^{\circ} 2$ ).

Respecto a la edad de los estudiantes, del $100 \%$ de los encuestados el $17.9 \%$ tienen entre 16 y 18 años, el 52.3\% tienen entre 19 y 21 años, el $25.1 \%$ tiene entre 22 y 24 años, el $3.4 \%$ tiene entre 25

\section{Gráfico $\mathrm{N}^{\circ} 1$}

Estudiantes de la Escuela de Negocios Internacionales de la UNMSM - Distribución por ciclos de estudio

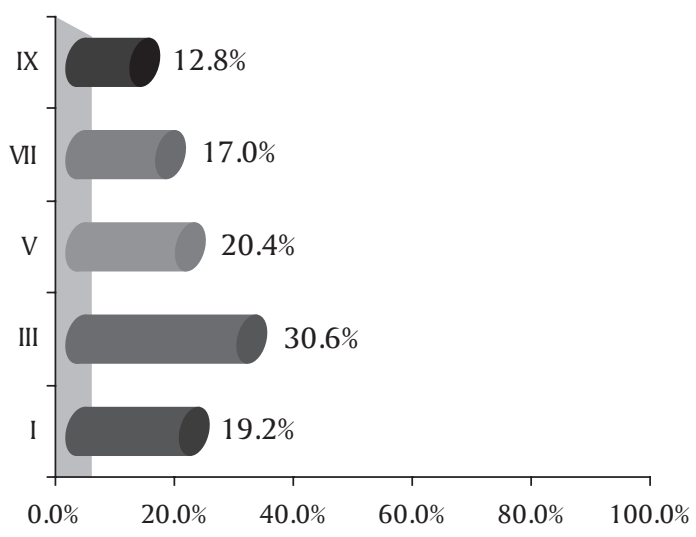

Gráfico $\mathrm{N}^{\circ} 3$

Estudiantes de la Escuela de Negocios Internacionales de la UNMSM - Distribución por edad

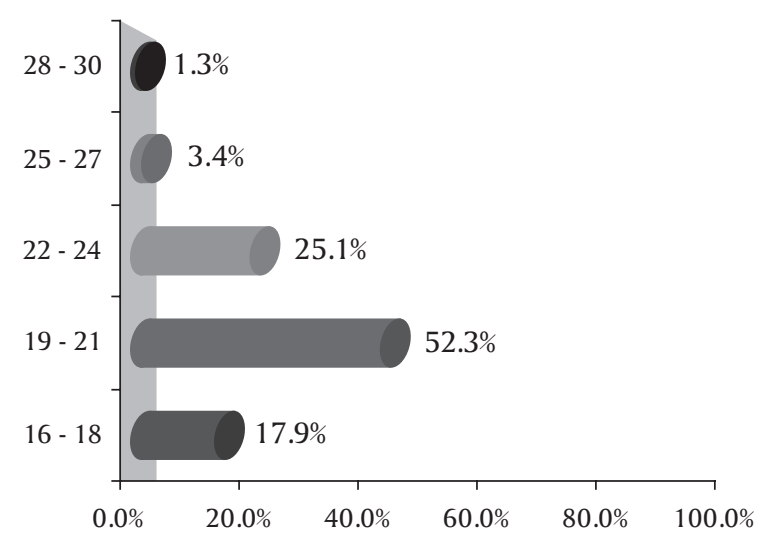

y 27 años y el $1.3 \%$ tiene entre 28 y 30 años (ver Gráfico N³).

Con relación al lugar de nacimiento de los encuestados el $68.1 \%$ han nacido en Lima, el $20 \%$ han nacido en provincia, el $7.7 \%$ han nacido en el Callao, el $1.2 \%$ han nacido en el extranjero y el $1.2 \%$ no responden (ver Gráfico $\mathrm{N}^{\circ} 4$ ).

Con respecto al lugar de residencia del $100 \%$ de los encuestados, el $32.8 \%$ pertenece al cono norte, un $28.9 \%$ pertenece al cono centro, un $21.7 \%$ al cono este, un $8.9 \%$ al cono Callao y un $7.7 \%$ al cono sur (ver Gráfico N ${ }^{\circ}$ ).

\section{Gráfico $\mathrm{N}^{\circ} 2$}

Estudiantes de la Escuela de Negocios Internacionales de la UNMSM - Distribución por sexo o género

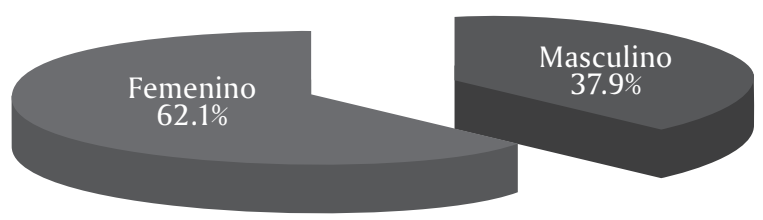

Gráfico $N^{\circ} 4$

Estudiantes de la Escuela de Negocios Internacionales de la UNMSM - Distribución por lugar de nacimiento

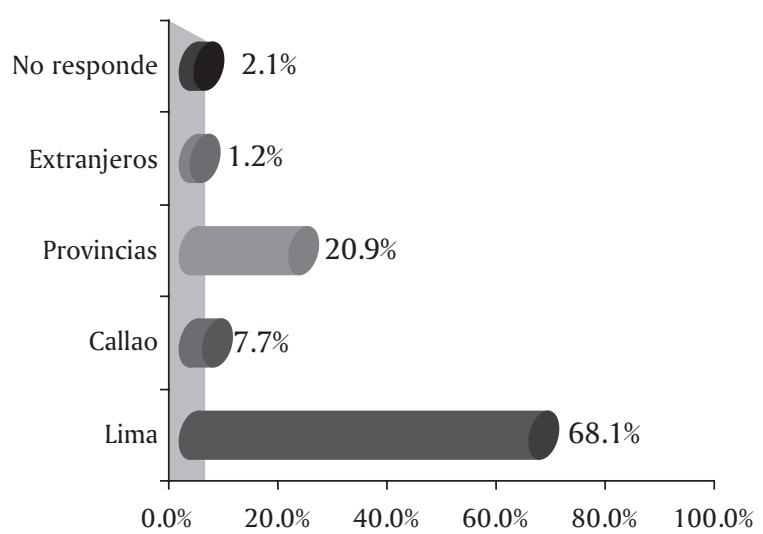


Del $100 \%$ de los estudiantes encuestados, el $83.4 \%$ tiene algún familiar con negocio propio y el $14.5 \%$ no lo tiene (ver Gráfico $\mathrm{N}^{\circ} 6$ ).

Del total de los encuestados, el 38.3\% tiene tíos con negocio, el $12.8 \%$ tiene padres con negocio propio (ver Gráfico N ${ }^{\circ}$ ).

Del $100 \%$ de los encuestados, el $85.1 \%$ no ha tenido empresa, sólo el $7.2 \%$ tiene o ha tenido empresa (ver Gráfico № 8).

Del 100\% de los encuestados, el $98.3 \%$ piensa formar una empresa y el $1.7 \%$ no ha pensado en formar una empresa (ver Gráfico $\mathrm{N}^{\circ}$ 9).

\section{Gráfico $N^{\circ} 5$}

Estudiantes de la Escuela de Negocios Internacionales de la UNMSM - Distribución por lugar de residencia

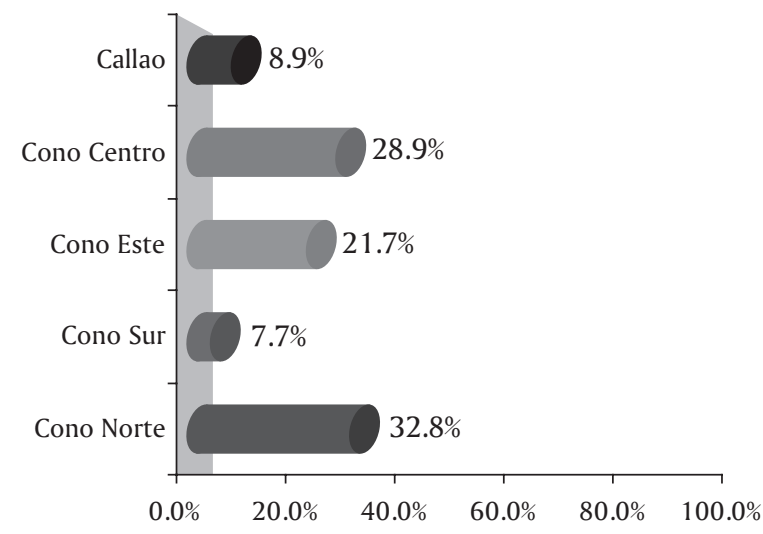

Gráfico $\mathbf{N}^{\circ} 6$

Estudiantes de la Escuela de Negocios Internacionales de la UNMSM - Familiares con negocio propio

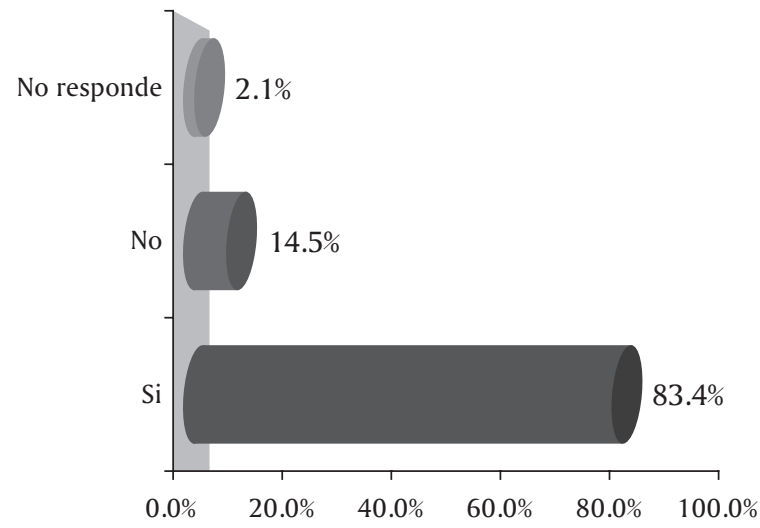

\section{DISCUSIÓN}

1. Los estudiantes de la especialidad de Negocios Internacionales de la Universidad Nacional Mayor de San Marcos poseen algún nivel de emprendedorismo, y este patrón se observa en el transcurso de los ciclos.

2. Existen diferencias significativas entre los valores motivacionales de los estudiantes de la especialidad de Negocios Internacionales de la Universidad Nacional Mayor de San Marcos que permiten afirmar que poseen actitud emprendedora.

\section{Gráfico $\mathrm{N}^{\circ} 7$}

Estudiantes de la Escuela de Negocios Internacionales de la UNMSM - Parentesco con familiar que tiene negocio

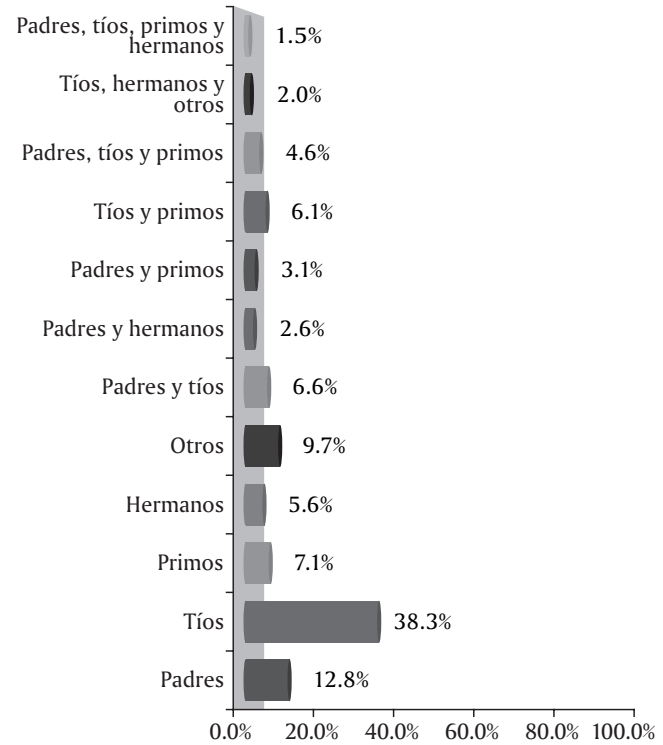

Gráfico $\mathrm{N}^{\circ} 8$

Estudiantes de la Escuela de Negocios Internacionales de la UNMSM - Tenencia de empresa

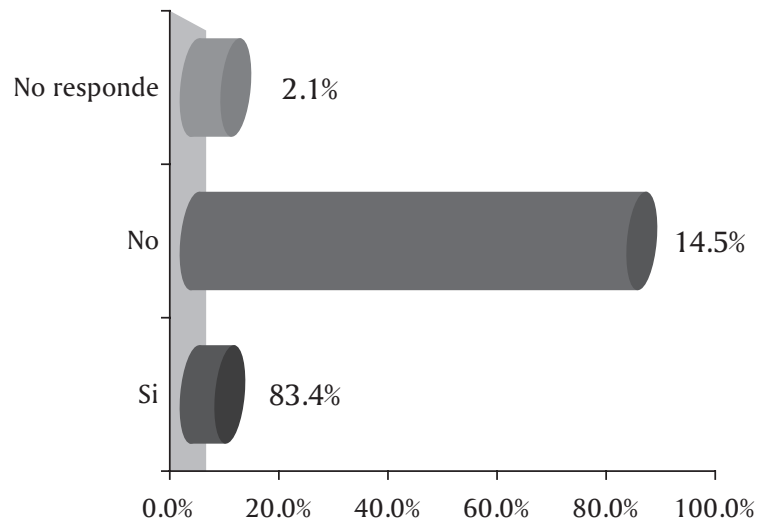




\section{Gráfico $\mathrm{N}^{\circ} \mathbf{9}$}

Estudiantes de la Escuela de Negocios Internacionales de la UNMSM - Intención de formar empresa (por ciclos)

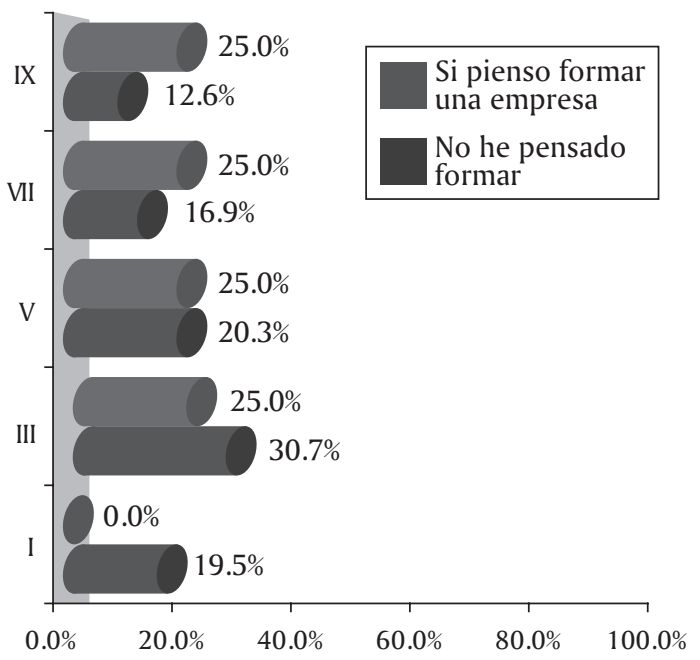

3. Los valores personales individualistas se relacionan en forma directa con los niveles de rasgos emprendedores, y los valores colectivistas se relacionan en forma inversa con los niveles de emprendedorismo.

4. En efecto, el emprendedor empresarial busca su satisfacción personal, de los suyos y posteriormente de los demás ya que, en la medida que al emprendedor le vaya bien, le irá bien a los demás. Por ello predominan los valores individualistas en relación directa con los rasgos emprendedores.

5. Los niveles de emprendedorismo se relacionan en forma directa con los valores ubicados en la dimensión individualista. Los estudiantes que poseen alto nivel de emprendedorismo se relacionan en forma directa con el valor poder, estimulación y autodirección, y los valores benevolencia, tradición y conformidad se relacionan en forma inversa con los niveles de emprendedorismo.

6. Los estudiantes hombres dan más importancia al valor poder que es un valor de la dimensión individualista, en comparación con las estudiantes mujeres. El poder significa obtener posición y prestigio social, control o dominio sobre las personas y recursos. La variable sexo influye ligeramente pero no es determinante en la estructura de los valores motivacionales, mientras que la edad no influye en la estructura de valores motivacionales, al igual que la condición laboral.

7. Con respecto al lugar de nacimiento de los estudiantes existen diferencias significativas: los estudiantes que han nacido en provincias presentan un promedio más alto de emprendedurismo en comparación con los de Lima y extranjeros.

8. Con respecto al análisis de los estudiantes que han tenido empresa, las puntuaciones más altas las alcanza el valor poder y, en los que no tienen empresa, el valor conformidad.

9. Los estudiantes que piensan formar empresas poseen puntuaciones más altas en el valor autodirección, pero también se observan puntuaciones altas en el valor universalismo. La explicación que encontramos es que al ser una universidad pública la tendencia se orienta hacia este valor. Los estudiantes que pertenecen a una familia donde el trabajo es por cuenta propia tienen mayor influencia en sus niveles de emprendedorismo, mientras que no existe relación entre las variables nivel de emprendedorismo y lugar de nacimiento; de la misma forma ocurre con la opción si ha tenido o tiene empresa.

\section{CONCLUSIONES}

1. Los resultados obtenidos en la presente investigación muestran que los estudiantes con algún nivel de emprendedorismo se identifican por valorar la independencia en el pensamiento y la toma de decisiones, éstas son características fundamentales para "emprender una empresa", destacando elementos como la creatividad, libertad, independencia, curiosidad y la elección de sus propias metas (valor de autodirección que corresponde a un valor de interés individualista); asimismo, valoran la comprensión, el aprecio, la tolerancia y atención hacia el bienestar de todas las personas y hacia la naturaleza (valor de universalismo que corresponde a un valor de interés mixto).

2. Los estudiantes de la E.A.P. de Negocios Internacionales de la Universidad Nacional Mayor de San Marcos se caracterizan por poseer niveles de emprendedorismo; este porcentaje es alto en el primer y tercer ciclo, en el quinto ciclo el porcentaje baja y luego sube en el séptimo y noveno ciclo. 
3. En los estudiantes de la E.A.P. de Negocios Internacionales de la Facultad de Administración de la Universidad Nacional Mayor de San Marcos el valor que prevalece es la autodirección, valor propuesto por Schwartz dentro de las dimensiones del individualismo, lo cual nos dice que los estudiantes de esta especialidad se caracterizan porque disfrutan con lo que hacen, tienen independencia en el pensamiento y eligen sus propias metas, por lo que poseen las características de ser emprendedores.

4. Los estudiantes hombres de la E.A.P. de Negocios Internacionales de la Facultad de Administración de la Universidad Nacional Mayor de San Marcos seleccionaron el valor poder como el más importante valor dentro de las dimensiones del individualismo; ello significa que los estudiantes hombres de esta especialidad buscan posición, prestigio social y ser reconocidos, en comparación con las estudiantes mujeres. En cuanto a la edad, no se observan diferencias significativas; tampoco en la condición laboral. Los estudiantes nacidos en provincia presentan un puntaje alto en el valor autodirección en comparación con los que nacieron en Lima y el extranjero. Los estudiantes del cono Callao presentan un puntaje alto en el valor hedonismo en comparación a los de los otros conos. Los estudiantes que en alguna oportunidad han tenido empresas alcanzan puntajes más altos en el valor poder en comparación con los que no tienen empresa.

5. Los estudiantes que piensan formar una empresa en el futuro presentan puntuaciones más altas en el valor autodirección en comparación con los estudiantes que no tienen en sus planes formar una empresa en el futuro.

6. Los niveles de emprendedorismo de los estudiantes de la especialidad de Negocios Internacionales de la Universidad Nacional Mayor de San Marcos se relacionan directamente con tener un familiar empresario. Podemos concluir que contar con familiares empresarios influyen significativa y positivamente en los niveles de emprendedorismo.

\section{BIBLIOGRAFÍA}

Alcarraz Rodríguez, Rafael. (2001). El emprendedor de éxito." Ciudad, Editorial Mc. Rally, 315 págs.
Alemán S., Luis. (2002). "Las PYMEs: evolución, análisis y perspectivas. Un enfoque financiero e integral”. En: Revista Oficial del Colegio de Contadores Públicos de Lima. Lima, noviembre 2002.

Assis Dornelas, José Carlos. (2001). Empreendedorismo. Transformando idéias en negócios. Rio de Janeiro, Campus.

Krushell, Jennifer. (2001). Solo para Emprendedores. Aproveche su juventud para crear un empresa de éxito. Lima, Ed. Norma, 306 págs.

Bernal T. César Augusto. (2000). Metodología de la investigación para administración y economía. Colombia, Prentice Educación de Colombia.

Formichella, Marta. (2004). El Concepto de Emprendimiento y su relación con la Educación, el Empleo y el Desarrollo Local. Buenos Aires, Editorial Aguilar.

Freyre, Andy. (2004). Pasión por Emprender. Lima, Ed. Aguilar, 239 págs.

Gouveia, Valdiney V. (2002). El Individualismo y el Colectivismo Normativo. Brasilia, Editorial Pionera.

Hair; Anderson; Tatham; y Black. (1999). Análisis Multivariante. Quinta Edición. Madrid, Pearson Educación.

Hernández, Roberto; Fernández, Carlos y Baptista, Pilar. (1991). Metodología de la Investigación. Segunda Edición. México, Mc Graw Hill.

Irigoyen, Horacio. (1997). PYMES, su economía y organización. Buenos Aires, Mc Graw Hill.

Jaramillo, Miguel y Parodi, Sandro. (2003). Jóvenes Emprendedores. Lima, Instituto Apoyo.

Moreano León, Juan Antonio. (2005). El Perfil Psicosocial del Emprendedor. Madrid, Consejo Económico Social (CES).

Sánchez C., Hugo y Reyes M., Carlos. (1985). Metodología y Diseños en la Investigación Científica . Lima, Editorial Aguilar.

Sánchez Yábar, Guido y Becerra F., S. (2005). Aprendiendo a Emprender. Lima, Artes y Diseños Láser.

Schwartz, S.H. (1990). "Individualism-Collectivism. Critique and proposed refinements". En: Journal of cross-cultural psychology. California, Sage Publication. Vol. 21, №2. 
Schwartz, S.H. (1992).Universals in the content and structure of value: Theoretical advances and empirical tests in 20 countries. En: Zanna, M.P. (ed). Advances in Experimental Social Psychology. New York, Academic Press. Vol. 25.

Sérida, Jaime. (2005). Global Entrepreneurship. Lima, Ediciones ESAN.

Sérida, Jaime; Borda, Armando; Nakamatsu, Keiko; Morales, Oswaldo y Yamakawa, Meter. (2006). Global Entrepreneurship Monitor.Lima, ESAN Ediciones.

Singelis, T.M. (1994). "The measurement of independent and interdependent self-construals”. En: Personality and Social Psychology Bulletin. Washington, APA.

Sonfield, Matthew y Lussier, Robert. (1995). "El Arte de emprender, cómo valorar las alternativas estratégicas del nuevo negocio”. En:
Revista Harvard Deusto Bussines. Vol. 16, № 82, febrero de 1995.

Triandis, H. (1994). "Recherches récentes sur l'individualism et le colectivism”. En: Les cahiers Internationaux de Psychologie Sociale. Washington, APA. Vol. 23.

Vallerand, R. J. (Ed.). (1994). Les fondements de la psychologie sociale. Québec, Gaëtan Morin.

\section{INTERNET}

www.uv.es/motiva/libromotiva/10MorianoSanchezP alaci.pdf.

Moriano, Juan A. (2005). Emprendedores en España, la República Checa y Bulgaria.En:jmoriano@ bec.uned.es.

Vinante, Luis José. (2004). Emprendedor: Creatividad - Resultados. En: www.iniciativasnet.com 